\title{
Surgical excision of a cardiac hydatid cyst from the right ventricle in a child
}

\author{
Alwaleed Al-Dairy ${ }^{1}$ and Rahim Abo Kasem ${ }^{1}$ \\ ${ }^{1}$ Damascus University Faculty of Medicine
}

June 6, 2021

\begin{abstract}
Cardiac Hydatid Cysts are uncommonly encountered entity of hydatid disease. Presentation may be with non-specific symptoms and sometimes with life threatening events. We present a rare case of a 9-year-old female who was diagnosed with a Cardiac Hydatid Cyst in the right ventricle, and underwent successful surgical excision
\end{abstract}

\section{Surgical excision of a cardiac hydatid cyst from the right ventricle in a child}

Alwaleed Al-Dairy ${ }^{1,[?]}$, MD, Rahim Abo Kasem².

${ }^{1}$ Assistant Professor in Cardiac Surgery at Faculty of Medicine, Damascus University, ${ }^{2}$ Medical student at Faculty of Medicine, Damascus University.

*Correspondence to :Alwaleed Al-Dairy: Assistant Professor inCardiac Surgery at College of Medicine, Damascus University, and Cardiac Surgeon at Damascus University Cardiac Surgery Hospital. Damascus, Syria.

Email: alwaleedaldairy.aa@gmail.com; Tel+963112133545, Fax +9632129437.

ORCID:Alwaleed Al-Dairy (0000-0002-2239-646X).

\begin{abstract}
Cardiac Hydatid Cysts are uncommonly encountered entity of hydatid disease, even in regions where hydatid cysts are endemic. Most patients are adults and asymptomatic. Presentation may be with non-specific symptoms such as dyspnea, chest pain, palpitations, cough, and sometimes with life threatening events. We present a rare case of a 9-year-old female who was diagnosed with a Cardiac Hydatid Cyst in the right ventricle, and underwent surgical excision.
\end{abstract}

Keywords : Cardiac Hydatid Cyst, Cystic Echinococcosis, Right Ventricle, cardiopulmonary bypass (CPB), Transthoracic echocardiography (TTE), Albendazole. .

\section{Key Clinical Message:}

Reporting the rare cardiac localization of hydatid disease in the right ventricle of a child, and underlining the importance of early diagnosis and treatment to avoid severe and life threatening complications.

\section{Introduction:}

Since time immemorial, human Echinococcosis has represented a serious disease burden of global concern $(1,2)$. It is a parasitic disease caused by the infection of larval stages of tapeworms belong to genus Echinococcus through the ingestion of food or water contaminated with their eggs, or by a direct contact with the 
animal hosts. The development of the larvae of Echinococcus Granulosus in humans (as accidental intermediate hosts) leads to Cystic Echinococcosis (CE) (Hydatidosis) (1,2,3). It is a hyperendemic disease in different regions of the world, significantly in rural areas $(1,4)$. The most frequently involved organs by hydatidosis are liver (more than $65 \%$ of cases) and lungs (both comprise at least $90 \%$ of cases) $(1,5)$. Cardiac hydatid disease is rare (0.5-2\% of all locations), and this may be due to the filtration of the embryos through the liver and lungs, and to the fact that cardiac rhythmic contractions prevent the cysts to implant within the cardiac wall. $(3,6)$. Clinical presentation of cardiac hydatid cysts is quite variable and depends on the age, size, location, and integrity of the cyst. In the majority of cases, patients are asymptomatic. Most common reported symptoms are dyspnea, chest pain, palpitations, and cough $(3,8,9)$. On the other hand, it may present with life threatening events such as anaphylactic shock, pulmonary embolism, acute coronary syndrome, or malignant arrhythmias due to subendocardial rupture which leads to the release of cyst contents into the blood stream. $(2,3,7)$. In endemic geographic areas, the most important issue regarding diagnosis is clinical suspicion. Transthoracic echocardiography (TTE) is the main diagnostic tool for cardiac hydatid disease. Computed tomography (CT) and magnetic resonance imaging (MRI) may provide additional diagnostic information $(2,3,7)$. Surgery is the treatment of choice in all cases to achieve higher survival rates with lower recurrence (1-10).

\section{Case presentation:}

A nine-year-old girl from northern province in Syria, presented to our hospital with recent developing exertional dyspnea. In her past medical history, she had undergone surgical resection of liver hydatid cysts four years ago. TTE showed a $50 \times 55 \mathrm{~mm}$ heterogeneous cystic mass localized in the free wall of the right ventricle outlet (figure 1). Routine laboratory tests were normal. Depending on TTE findings, the patient's demographic origin, and her surgical history, the diagnosis of right ventricle hydatid cyst was established. After a 5-day oral Albendazole course, surgery was performed through median sternotomy, and cardiopulmonary bypass (CPB) was prepared. The cyst was apparent within the wall of the right ventricle outlet (figure 2 ). The operating field was isolated by medical gauze saturated with hypertonic saline solution. Without arresting the heart, a small incision on right ventricular outlet was carried out. The contents of the cyst were aspirated, and the cyst cavity was injected with $30 \%$ saline solution. Then the cyst was dissected and enucleated (Figure 3). The patient recovered without complications, and was discharged home six days later on albendazole for 12 weeks.

\section{Discussion:}

CE is highly prevalent in southern and northern provinces of Syria; however, epidemiologic studies on human echinococcosis are still not available (11). Our patient was from northeastern province in Syria, which is notably endemic for CE. In rare cases, the larvae reach the heart mainly through the coronary arteries and develop into hydatid cysts. Young adults are the most frequently affected, but very rare cases of cardiac hydatid cysts in children have been reported. Since it receives the most blood supply, the left ventricular wall is most frequently involved (55-60\%), whereas the right ventricle is less involved (10-15\%). Our case is a rare presentation of cardiac hydatid cyst within the right ventricle in a child. It has been reported that in cases of right ventricle hydatid cysts, intracavitary rupture is more frequent, and may lead to pulmonary embolization, pulmonary hypertension, and death $(2,3,9,10)$. To avoid these complications, it is crucial to make an urgent diagnosis and investigations. High index of suspicion was the key of diagnosis in our case (the geographic origin and surgical history). There are multiple laboratory tests, such as Casoni's intradermal test, the Weinberg reaction, and peripheral blood eosinophil count; however, they are not reliable since they carry false negative results (7). Electrocardiogram and Chest radiograph are not specific (7). TTE is the preferred diagnostic modality in detecting the cysts and their location and size $(3,7)$. Other highly useful modalities include CT and MRI, which can be informative for a better surgical approach $(3,5,7)$. The surgical excision combined with Albendazole therapy is still the gold standard for treatment of cardiac hydatid cysts (1-10). It is possible to excise the superficially or epicardially located cysts without the use of CPB; however it is more recommended to use CPB as it shows higher safety and less risk of cyst contents leakage during surgery $(7,8)$. In order to reduce the cyst tension and avoid an accidental rupture, the entire cyst contents must be 
aspirated. Moreover, the cyst must be injected with protoscolicidal agent to create an osmotic gradient that kills the protoscolices to avoid any possible recurrence in case of their spillage or locally invasion during the operation. For this purpose, packing the operative field with gauze and sponges soaked in a scolicidal agent is performed. we used hypertonic saline solution due to its effectiveness and relatively non-toxicity $(8,13)$. Albendazole ( $400 \mathrm{mg}$ twice daily) should be prescribed at least 4 days preoperatively, and continued for 12 weeks postoperatively $(2,3,8,9)$. The effectiveness of other therapeutic techniques such as puncture, aspiration, injection of a scolicidal agent, reaspiration, and radiofrequency ablation in the treatment of cardiac hydatid cysts are still unclear (8).

\section{Conclusion:}

Cardiac hydatid cyst is very uncommon disease, rarely affecting the right ventricle in children. The clinical course varies widely, and the presentation may be insidious with persistent potential risk of life threatening events. CE must be suspected in endemic areas, diagnosed with appropriate imaging techniques, and treated appropriately.

\section{Figure legends:}

Figure 1: TTE image of the cyst (The Star) in the right ventricle in four chamber view.

Figure 2: Intraoperative image showing the swelling in the right ventricle outlet (The Star) which refers to the cyst.

Figure 3: Image of the excised cyst.

\section{Author Contribution}

Alwaleed Al-Dairy : Planned and performed the work leading to the report. Wrote and reviewed successive versions and participated in their revisions.

Rahim Abo Kasem : wrote and reviewed the successive versions and participated in their revisions

Author's Statement:

Consent for publication was granted by the patient's mother.

\section{Conflict of interest:}

The authors have no conflict of interest

\section{Funding information:}

The authors declare that there is no funding resources

\section{Acknowledgement: None}

\section{Data Availability Statement}

The data that support the findings of this study are available from the corresponding author, [A.A], upon reasonable request.

\section{References:}

1-Eckert J, Gemmell M A, Meslin, François-Xavier, PawlowskiZ. S \& World Health Organization. ( 2001): WHO/OIE manual on echinococcosis in humans and animals : a public health problem of global concern / edited by J. Eckert ... [ et al.] . Paris, France :World Organisation for Animal Health. https://apps.who.int/iris/handle/10665/42427

2- Oraha AY, Faqe DA, Kadoura M, Kakamad FH, Yaldo FF, Aziz SQ: Cardiac Hydatid cysts; presentation and management. A case series. Ann Med Surg (Lond). 2018 Apr 7;30:18-21. 
3- Jamli M, Cherif T, Ajmi N, Besbes T, Mgarrech I, Jerbi S, Kortas C, Tarmiz A: Surgical Management and Outcomes of Cardiacand Great Vessels Echinococcosis: A 16-Year Experience. Ann Thorac Surg. 2020 Oct;110(4):1333-1338.

4- Noaman H, Rawaf S, Majeed A, Salmasi AM: Hydatid Cyst of the Heart. Angiology. 2017 Oct;68(9):765768 .

5- Dursun M, Terzibasioglu E, Yilmaz R, Cekrezi B, Olgar S, Nisli K, Tunaci A: Cardiac hydatid disease: CT and MRI findings. AJR Am J Roentgenol. 2008 Jan;190(1):226-32.

6- Elkarimi S, Ouldelgadia N, Gacem H, Zouizra Z, BoumzebraD, Blelaabidia B, Elhattaoui M: Tamponnaderévélant un kystehydatique intra-péricardique - un cas [Tamponade reveals an intra-pericardial hydatid cyst - a case report]. Ann CardiolAngeiol (Paris). 2014 Sep;63(4):267-270. French.

7. Yaliniz H, Tokcan A, Salih OK, Ulus T: Surgical treatment of cardiac hydatid disease: A report of 7 cases. Tex Heart Inst J. 2006;33(3):333-339.

8- Wadhawa V, Shah J, Doshi C, Ramani J, Lakhia K, Rathod D, Tavar R, Kothari J: Surgical overview of cardiac echinococcosis: arare entity. Interact CardiovascThorac Surg. 2018 Aug 1;27(2):191-197.

9- Pakis I, Akyildiz EU, Karayel F, Turan AA, Senel B, Ozbay M, Cetin G: Sudden death due to an unrecognized cardiac hydatid cyst:three medicolegal autopsy cases. J Forensic Sci. 2006 Mar;51(2):400-2.

10- Ipek G, Omeroglu SN, Goksedef D, et al. Large cardiac hydatid

cyst in the interventricular septum. Tex Heart Inst J. 2011;38:719-722.

11- Sadjjadi SM: Present situation of echinococcosis in the Middle East and Arabic North Africa. Parasitol Int. 2006;55 Suppl:S197-202.

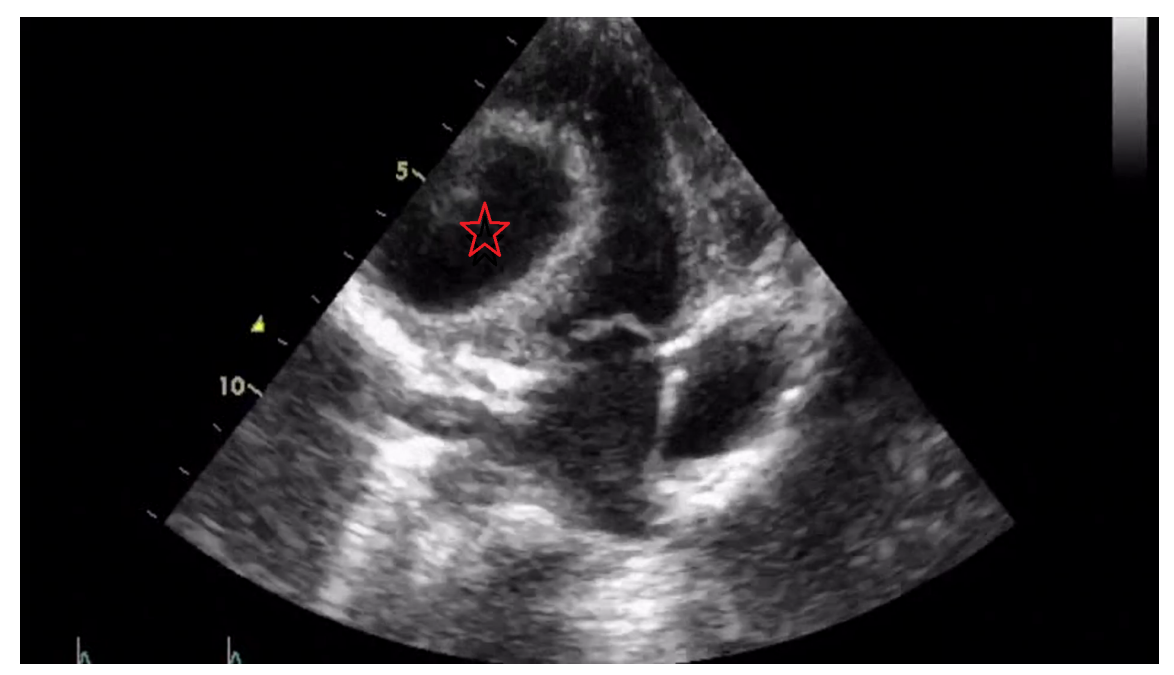



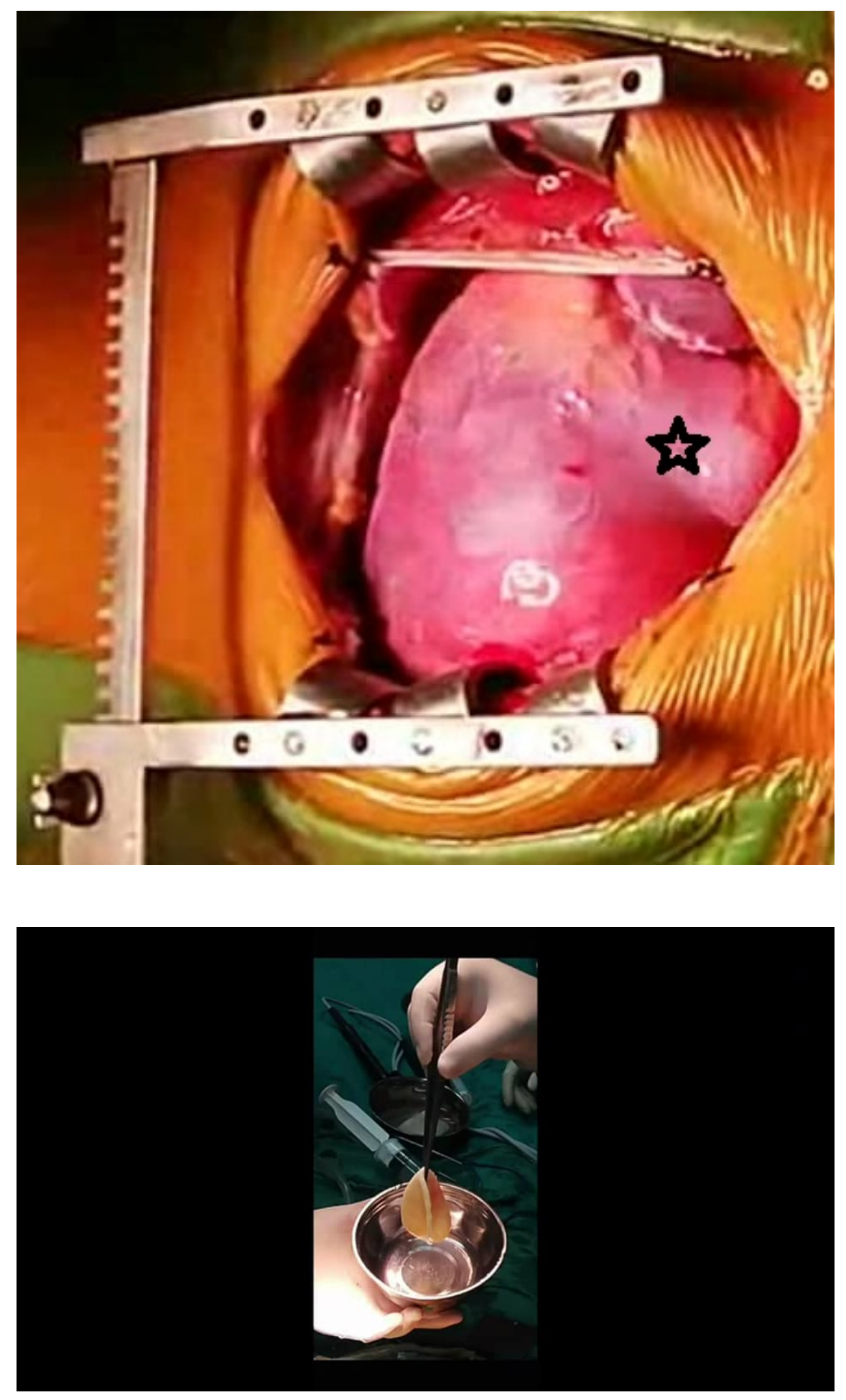